\title{
Application of Algorithm of Smoothing Pseudo-range with Carrier Phase to DRTS System
}

\author{
Hong-jiao MA ${ }^{1,2, *}$, Fei-fei YAN ${ }^{1,2,3}$ and Zhi-jun $\mathrm{HOU}^{1,2,3}$ \\ ${ }^{1}$ National Time Service Center, Chinese Academy of Sciences, Xi'an 710600, China \\ ${ }^{2}$ Key Laboratory of Time and Frequency Primary Standards, National Time Service \\ Center, Chinese Academy of Sciences, Xi'an 710600, China \\ ${ }^{3}$ University of Chinese Academy of Sciences, Beijing 100039, China
}

Keywords: Dual one-way ranging, Carrier phase, Code phase.

\begin{abstract}
The algorithm of smoothing pseudo-range with carrier phase and its application to the dual ranging and time synchronization (DRTS) system are introduced. The calculation and analyses of the measured data show that the application of the algorithm of smoothing pseudo-range with carrier phase to the DRTS can improve the ranging accuracy of DRTS. The processing methods for the pseudo-code mistakes and carrier phase leaps which occurred during the measurements and also given in this paper, and the simulation and analyses show that these methods can reduce the error caused by the pseudo-code mistakes and carrier phase leaps.
\end{abstract}

\section{Introduction}

The realization of Aerospace integration, together with the high accuracy time synchronization measurement and control platform, needs DRTS system to provide high accuracy time synchronization service for them. DRTS system can obtain respective relative pseudo range by signal transmitter and receiver, which mounted on two ranging terminals, performing pseudo code measurement and carrier phase measurement. In this ranging method, since the transmitting path and the receiving path of ranging signal are substantially the same but in the opposite direction, thus this ranging mode can eliminate the error caused by the difference between transmitting paths as much as possible, thus precise ranging and time synchronization between these two ranging terminals can be achieved ${ }^{[1-4]}$. However, the pseudo range measurements obtained through pseudo code ranging contains a considerable amount of measurement noise, which lowers the accuracy of ranging; and although the pseudo range measurements obtained through carrier phase ranging has relatively high measurement accuracy, it also has integer ambiguity and cycle slip of phase. Therefore, the accuracy of DRTS system can be greatly improved if using the high accuracy carrier phase ranging measurements to smooth the pseudo code ranging measurements.

\section{The Principle of Carrier Phase Smoothing}

Carrier phase smoothing is a high accuracy ranging method which combines the respective advantages of pseudo code ranging and carrier phase ranging. The ranging equations of pseudo code ranging and carrier phase ranging can be expressed as follows:

$$
\rho=R+\varepsilon_{\rho}
$$




$$
\lambda(\phi+N)=R+\varepsilon_{\phi}
$$

Wherein $\rho$ represents pseudo range measurement value measured by ranging terminals, $R$ represents the real distance between terminals, ${ }^{\varepsilon_{\rho}}$ is the error during pseudo code ranging, $\lambda$ represents wave length of the carrier, $\phi$ represents phase of the carrier, represents integer ambiguity in carrier phase ranging, which stays the same if there is no cycle slip of phase occurs. ${ }^{\varepsilon_{\phi}}$ represents the error during carrier phase ranging. If ${ }^{\varepsilon_{\rho}}$ and ${ }^{\varepsilon_{\phi}}$ are ignored, and assume that the random error during ranging obeys Gaussian distribution, then the random error can be reduced through Statistical Methods. Assume that $\mathrm{n}$ continuous observation has been carried out, then the measurement equation can be expressed as:

$$
\left\{\begin{array}{l}
\rho_{t 1}=\lambda\left(\phi_{t 1}+N\right) \\
\rho_{t 2}=\lambda\left(\phi_{t 2}+N\right) \\
\mathrm{M} \\
\rho_{t n}=\lambda\left(\phi_{t n}+N\right)
\end{array}\right.
$$

The following equation can be obtained by performing $n$ times of iteration on the measurement equation described above:

$$
\begin{aligned}
& \lambda N=\frac{1}{n} \sum_{k=1}^{n}\left(\rho_{t k}-\lambda \phi_{t k}\right) \\
& \bar{\rho}_{t n}=\lambda \phi_{t n}+\frac{1}{n} \sum_{k=1}^{n}\left(\rho_{t k}-\lambda \phi_{t k}\right)
\end{aligned}
$$

Wherein equation is a pseudo range equation after performing carrier phase smoothing. Since the random error during carrier phase ranging is much less than the random error during pseudo code ranging, that is to say $\varepsilon_{\phi}=\varepsilon_{\rho}$, therefore, according to equation (9) and Error propagation principle, the following equation can be obtained:

$$
\delta_{\rho}^{2}=\varepsilon_{\phi}^{2}+\frac{1}{n}\left(\varepsilon_{\rho}^{2}+\varepsilon_{\phi}^{2}\right) \approx \frac{1}{n} \varepsilon_{\rho}^{2}
$$

Wherein $\delta_{\rho}$ represents the pseudo range error after being smoothed by carrier phase. As can be seen from the above equation, after performing $n$ times of smoothing, the amount of smoothed pseudo range error has been reduced to approximate $1 / \sqrt{n}$ of the original amount, which shows that after the carrier phase smoothing processing, the random error of code phase measurements have been effectively suppressed. If the parameter $\mathrm{n}$ is big enough, then the accuracy of pseudo range measurement will be substantially increased, which ranges in the accuracy of pseudo code ranging and the accuracy of carrier phase ranging.

\section{Application of Carrier Phase Smoothed Pseudo Range Algorithm in DRTS}

\section{DRTS Carrier Phase Smoothed Pseudo Range Algorithm}

DRTS system needs to update data in real time, thus carrier phase smoothed pseudo ranging needs to adopt real-time smoothing mode. However, in the process of carrier 
phase smoothed pseudo ranging mentioned above, the smoothed results will be obtained after measuring and storing $\mathrm{n}$ times of measurement data, which does not meet the real-time characteristics for the carrier phase smoothed pseudo ranging required by dual ranging and time synchronization system. Therefore, combined with the real-time requirement by dual ranging and time synchronization system, DRTS carrier phase smoothed pseudo range algorithm is designed, that is, deriving a pseudo range observation at a certain time through the use of pseudo range observations and carrier phase observations at multiple epochs. According to equation (9), the real-time expression of carrier phase smoothed pseudo range can be expressed as follows by applying the recursive methods thereto:

$$
\bar{\rho}_{t n}=\lambda \phi_{t n}+\frac{1}{n} \sum_{k=1}^{n}\left(\rho_{t k}-\lambda \phi_{t k}\right)=\lambda \phi_{t n}+\frac{1}{n}\left(\rho_{t n}-\lambda \phi_{t n}\right)+\frac{n-1}{n}\left(\bar{\rho}_{t n-1}-\lambda \phi_{t n-1}\right)=\frac{1}{n} \rho_{t n}+\frac{n-1}{n}\left(\bar{\rho}_{t n-1}+\lambda \phi_{t n}-\lambda \phi_{t m-1}\right)
$$

Wherein $\bar{\rho}_{t n}$ is the smoothed result at th after performing carrier phase smoothed pseudo ranging, $\mathrm{n}$ represents the smoothing times, which determines the degree of dependency that the smoothed pseudo range to the change amount of carrier phase, thus a bigger $\mathrm{n}$ means a smoother results. Fig.1 illustrates the flowchart of DRTS carrier phase smoothed pseudo range algorithm.

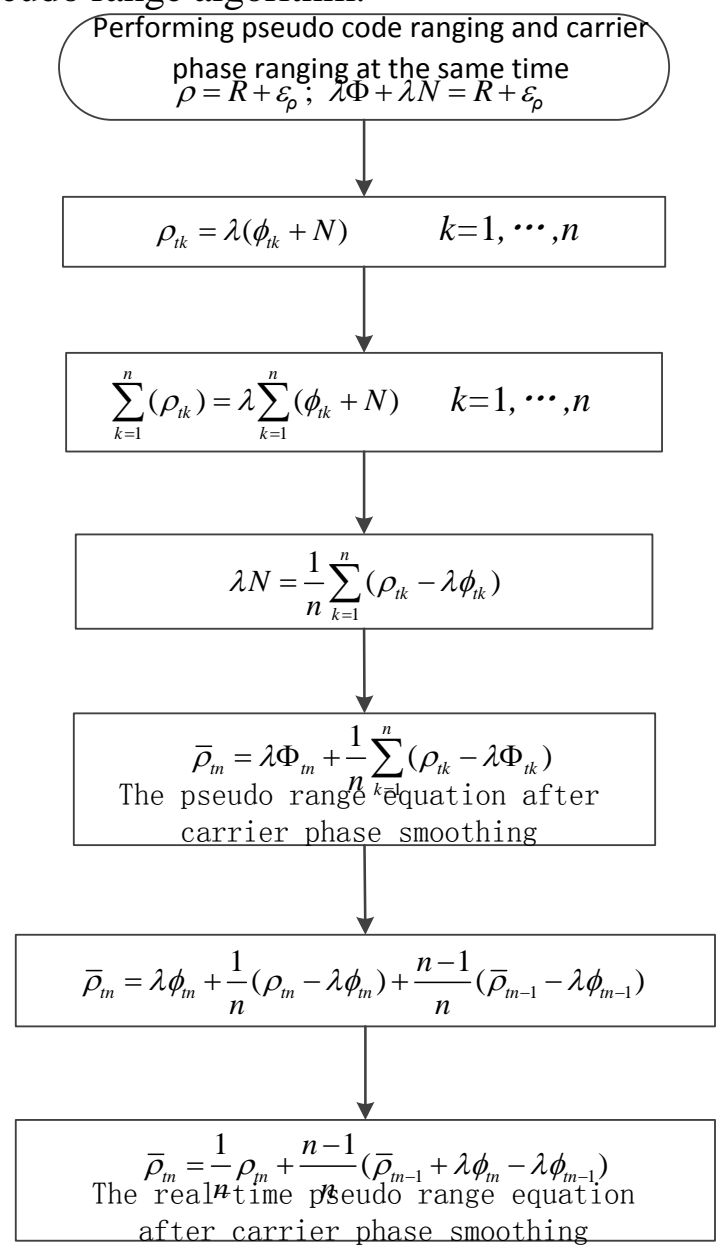

Fig.1 the real-time equation of carrier phase smoothed pseudo range

\section{Noise Analysis of Carrier Phase Smoothed Pseudo Range}

In order to analyze the pseudo range noise of DRTS carrier phase smoothed pseudo range algorithm, Symmetrical cables of equal length of $100 \mathrm{~m}$ is used to simulate the space link, and serial communication port of DRTS systems is used to collect pseudo 
range data and carrier phase data, as the measured data of this simulation experiment. In this experiment, 3300 data between 09:00:00 and 09:55:00 on March 31, 2014, which is collected at each $1 \mathrm{~s}$ intervals, is selected as measured data. According to the process in Fig.1, MATLAB simulation software is used to analyze the pseudo range noise of DRTS carrier phase smoothed pseudo range algorithm, wherein the said pseudo range noise obtained by pseudo range noise generation module written in MATLAB. Fig. 2(a), 2(b), 2(c) and 2(d) respectively show the comparison of initial pseudo range noise and smoothed pseudo range noise when the smoothing times M equals to 10, 100, 200 and 300 .

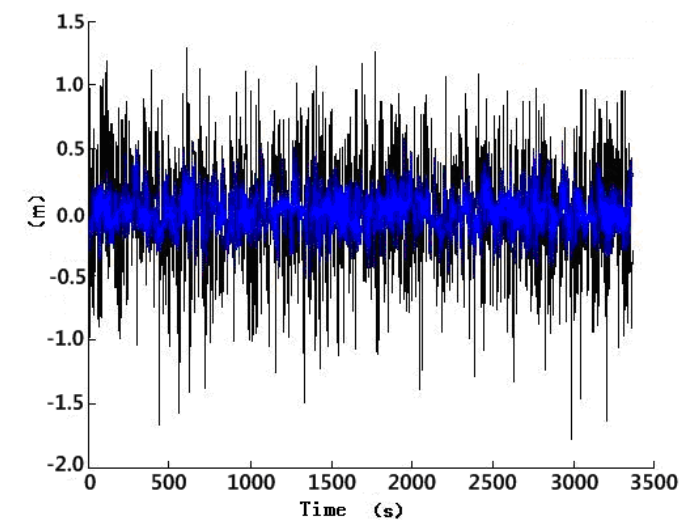

Fig. 2(a) Smoothing times $M=10$

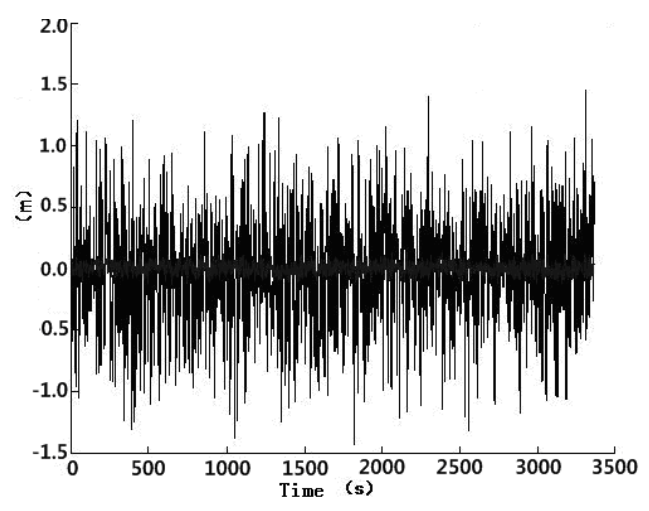

Fig. 2(c) Smoothing times $M=200$

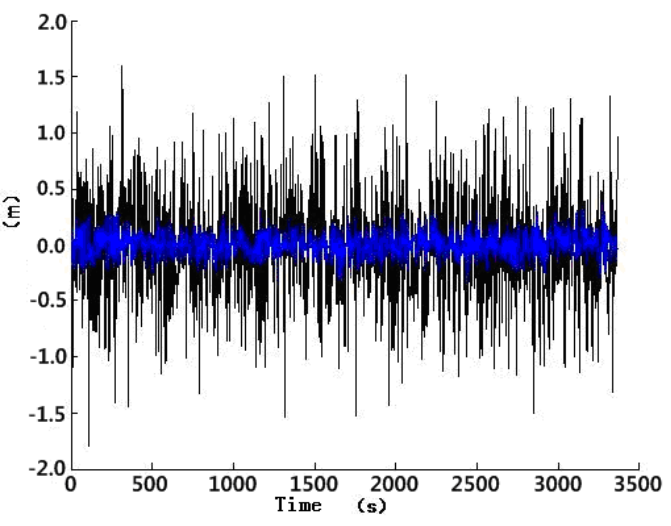

Fig. 2(b) Smoothing times $M=100$

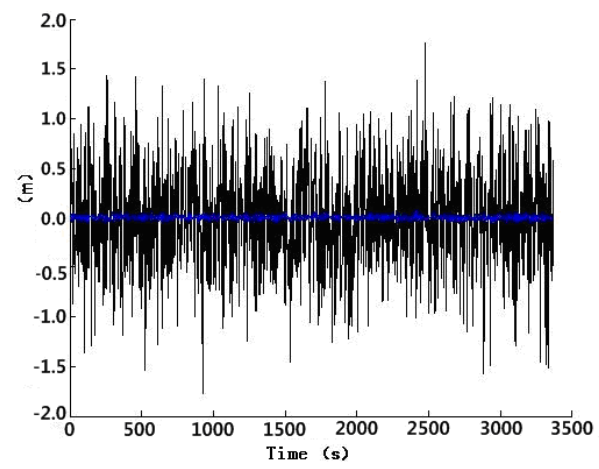

Fig. 2(d) Smoothing times $M=300$

\section{Conclusion}

Simulation results show that DRTS carrier phase smoothing algorithm can improve the ranging accuracy of dual ranging and time synchronization system, and the method of repairing pseudo code error and carrier phase cycle slip phenomenon can reduce the ranging error to a certain extent, then ensuring the measurement accuracy of the system.

\section{References}

[1] MA Hong-Jiao, WU Hua-Bing, et al, Design and analysis of system of dual ranging and time synchronization, Journal of time and Frequency, no. 01, pp. 18-24, 2014.

[2] KOUD. Analysis of the Characteristics of the Grace Dual One-Way Ranging System [D]. USA: the University of Texas at Austin, 2008: 161. 
[3] PAN Shu-guo, WANG Qing, KE Fu-yang, et al. Method for integer ambiguity resolution in GPS network RTK[J]. Journal of Southeast University, 2009, 25(4): 491-495.

[4] SA-TIM-MA-1004. SATRE Modem Overview [K]. 2002. 